\title{
Desnaturalización del dispositivo pedagógico en la educación del cuerpo
}

\author{
Denaturalization of the pedagogic device in body \\ education
}

\section{Desnaturalização do dispositivo pedagógico na educação do corpo}

\begin{abstract}
María Valeria Emiliozzi*
Resumen: En este escrito trataremos de plantear las relaciones entre Pedagogía y Educación en general, y con la Educación Física en particular, ya que el discurso pedagógico continúa operando como un saber que fundamenta el curriculum de la Educación Secundaria (Argentina). Se buscará desplegar un análisis sobre el saber y las relaciones de poder que permitieron las condiciones de posibilidad de la pedagogía, y la conformación de un modo de concebir al sujeto a partir de la sustancia, de lo tangible. La problematización del sujeto, implica un giro epistemológico que posee consecuencias en el modo de pensar las relaciones entre teoría y práctica, enseñanza y aprendizaje, profesor y alumno, entre otras; y abre un camino que no pretende ser algo verdadero, en el sentido de definitivo, sino otra herramienta.
\end{abstract}

Palabras claves: Sujeto. Educación. Pedagogía.

\begin{abstract}
This paper addresses the relationship between pedagogy and education in general, and Physical Education in particular, as the pedagogical discourse continues to operate as a sort of knowledge underpinning the curriculum of secondary education (Argentina). The study seeks to deploy an analysis of knowledge and power relations that allowed the conditions for the possibility of pedagogy, and gave rise to a conception of the subject from the substance, the tangible. The problematization of the subject involves an epistemological shift that has consequences for the way of thinking the relationship between theory and practice, teaching and learning, teachers and students, among others; and opens a path that does not intend to be something real, in the final sense, but another tool.
\end{abstract}

Keywords: Subject. Education. Educational.

\footnotetext{
*Doctoranda en Ciencias de la Educación por la UNLP. Ayudante Diplomada en la cátedra Educación Física 5 correspondiente al profesorado en Educación Física (FaHCE-UNLP). Becaria doctoral del CONICET. Investigador Categoría V del Programa de Incentivos de la Nación. E-mail: <val_emiliozzi@hotmail.com>
} 
Resumo: Neste artigo busco expressar a relação entre a pedagogia e a educação em geral e da Educação Física em particular, uma vez que o discurso pedagógico continua a operar como um conhecimento que sustenta o currículo do ensino secundário (Argentina). O artigo busca implementar uma análise de conhecimentos e relações de poder que permitiu que as condições de possibilidade da pedagogia e da formação de uma concepção do sujeito a partir da substância, do tangível. A problematização do tema envolve uma mudança epistemológica que tem consequências na forma de pensar a relação entre teoria e prática, ensino e aprendizagem, professores e alunos, entre outros; e abre um caminho que não tem a pretensão de ser algo real, no sentido final, mas outra ferramenta.

Palavras-chave: Sujeito. Educação. Pedagogia.

\section{Introducción}

La Educación se constituye a partir de un saber pedagógico que conforma los modos de gobierno sobre el cuerpo que se sostienen hasta el presente. En la Ley de Educación Nacional N²6.206 del año 2006, y la Ley de Educación Provincial No 13.688 de Buenos Aires del año 2007, "resulta imprescindible articular y explicitar en un Marco General de Política Curricular las bases pedagógicas que orientan las decisiones de los Niveles y Modalidades en lo relativo a las definiciones curriculares y la organización institucional" (DGCyE, 2007, p. 9). Esto implica analizar la educación con un dominio de saberes y con un conjunto de prácticas normativas, ya que la experiencia por la cual debe pasar el alumno en la Educación del cuerpo debe estar enmarcada en "condiciones pedagógicas para que el grupo se constituya en un entramado de relaciones humanas sostenedoras del aprendizaje motor" (DGCyE, 2006b, p. 133), y en la educación en general como "propuesta pedagógica que posibilite construir proyectos de futuro" (DGCyE, 2010, p. 5).

Sostener la educación sobre las bases de la pedagogía implica relaciones entre figuras epistemológicas o una teoría pedagógica que dé lugar a un saber que tome el estatuto y el papel de la ciencia. Por ello, reconocer la contingencia y la historicidad de esta base nos permite tomar un punto de vista arqueológicogenealógico $^{1}$ para analizar la pedagogía, no como un espacio neutro o de mediación para el desarrollo del alumno, sino como una práctica que produce formas de experiencia sobre el sujeto de un modo particular.

Según el diccionario de la Real Academia Española pedagogía, refiere a "ciencia que se ocupa de la educación y la enseñanza". En el mismo sentido,

\footnotetext{
${ }^{1}$ Este eje de la investigación toma su sentido de los estudios de Michel Foucault.
} 
Kant (2003, p. 45) establece que "la pedagogía o teoría de la Educación es física o práctica; en cuanto física estudia los cuidados -que comparte el aprendizaje animal con el humano-; en cuanto práctica o moral su estudio es relativo al comportamiento". Definir a la pedagogía como una teoría no refiere a una aplicación práctica, sino que es considerarla una práctica en sí misma (Cf. Foucault, 1992). La teoría no es comprendida como un conjunto de determinaciones que se imponen desde el exterior al pensamiento de los individuos, sino que "constituye más bien el conjunto de las condiciones según las cuales se ejerce una práctica" (Foucault, 2008a, p. 270). El discurso pedagógico se sostiene en un saber que tiene una historia, una serie de experiencias colectivas racionales que responden a un conjunto de reglas que construyen un modo de definir al sujeto de la educación. Foucault (2009a, p. 387-388) dice: "llamemos 'pedagógica', si quieren, la transmisión de una verdad que tiene la función de dotar a un sujeto cualquiera de aptitudes, capacidades, saberes, etcétera, que no poseía antes y que deberá poseer al término de esa relación pedagógica".

¿Qué efectos de verdad produce la pedagogía? El telón de fondo que crea las condiciones de existencia de la pedagogía, y de los sistemas educativos en general, es un proceso con el que comienza la reorganización de nuestra vida en el mundo, que da lugar a nuestra moderna vida urbana. Por ello, la pedagogía y la constitución del sistema educativo argentino responden a una matriz sociopolítica en la que el Estado define los parámetros de socialización e incorporación cultural de la población (cf. Tiramonti, 2007, p. 17). Pero, para analizar esta constitución, es necesario desplegar las relaciones que le sirven de soporte a la pedagogía, no en las formas en que la verdad se manifiesta, sino en las que se produce.

\section{Gobierno y pedagogía}

$\mathrm{Al}$ deshacerse las estructuras feudales, se produce un movimiento que introduce el problema del gobierno en relación con los grandes territorios: ¿cómo gobernar?, ¿cómo gobernar de la mejor manera posible?, ¿cómo fortalecer al Estado? En el sentido que le otorga Foucault (2007), gobernar, según el principio de razón de estado, refiere a actuar con el objetivo de hacer fuerte al Estado, ante cualquier amenazada que pueda destruirlo. De esta manera, el Estado se estructura de acuerdo a cierto deber ser que le permita constituirse de esta manera razonada y calculada. Ahora bien, ante el interrogante de cómo llevar la recta disposición de ese Estado, la pregunta que se antepone es cómo gobernar de la mejor manera posible, lo cual produce un corte espacio-temporal concreto entre dominios de saber, tipos de normatividad y formas de subjetivación. "Una correlación de ese tipo es la que se puede encontrar, también en un corte espacio-temporal particular, la estructura y el funcionamiento de un dispositivo pedagógico" (LARROSA, 1995, p. 289). 
$\mathrm{Al}$ abrir la puerta hacia la literatura sobre el gobierno, nos encontramos con un espíritu que anima un tipo de debate político aún hasta el siglo XVI y con otro muy distinto del siglo XVI en adelante. En un primer momento lo distintivo del príncipe, apoyado en la literatura El príncipe de Maquiavelo, se sostiene bajo el lema de que la soberanía de un Estado se puede mantener por la relación de singularidad y exterioridad del príncipe, cuya función es proteger y fortalecer su principado (asignado por herencia o por conquista). En cambio, para la literatura antimaquiaveliana el arte de gobernar está compuesto de prácticas múltiples que abarcan al padre de familia, al pedagogo, entre otros, y que van más allá de la relación singular del príncipe. ${ }^{2}$ Aquí se distinguen tres formas de gobierno, cada una de las cuales depende de una forma de ciencia o reflexión específica: "el gobierno de sí mismo, que depende de la moral; el arte de gobernar una familia como se debe, que depende de la economía; y, por último, la ciencia de gobernar bien el Estado, que depende de la política" (FOUCAULT, 2009b, p. 118).

El advenimiento de una profunda literatura indica, en realidad, una reactivación del interrogante sobre cómo gobernar, que se efectúa en el marco sociopolítico que mencionábamos, acompañado de dos movimientos: uno ligado a una concentración estatal (la aparición de los estados nacionales), y otro a la dispersión y escisión religiosa (la Reforma).

Desde luego, tanto en la Edad Media como en la Antigüedad grecorromana nunca faltaron esos tratados que se presentaban como consejos al príncipe en cuanto a la manera de conducirse, de ejercer el poder, de conquistar la aceptación o el respeto de los súbditos; consejos para amar a Dios, obedecer a Dios, hacer regir en la ciudad de los hombres la ley de Dios, etc. Pero creo que lo sorprendente, y bastante, es que a partir del siglo XVI y en todo el período que va, a grandes rasgos, de mediados de ese siglo hasta fines del siglo XVIII, vemos el desarrollo y florecimiento de una serie muy considerable de tratados que ya no se muestran exactamente como consejos al príncipe y tampoco, aún, como ciencia de la política, se presentan como artes de gobernar. Me parece que, en términos generales, el problema del 'gobierno' estalla en el siglo XVI, de manera simultánea, acerca de muchas cuestiones diferentes y con múltiples aspectos. El problema, por ejemplo, del gobierno de sí mismo. El retorno al estoicismo gira, en el siglo XVI, alrededor de esta reactualización del problema: como gobernarse a sí mismo. El problema, igualmente, del gobierno de las almas y las conductas, que fue, claro está, todo el problema de la pastoral católica y protestante. El problema del gobierno de los niños, y aquí está la gran problemática de la pedagogía, tal como aparece y se desarrolla en el siglo XVI. (FOUCAULT, 2009b, p. 110).

\footnotetext{
${ }^{2}$ En esta literatura encontramos la obra de G. de La Perrière (Le Miroir politique, contenant diverses manières de gouverner et policer les républiques, 1555) y François de la Mothe Le Vayer (L'œconomie du Prince, 1653).
} 
Este interrogante, entonces, deja ver dos formas, una doble continuidad: una continuidad ascendente (quien gobierne el Estado tiene que poder gobernarse a sí mismo) y una continuidad descendente (en un Estado bien gobernado, los padres saben gobernar la casa y por ende los individuos se comportan correctamente). Estas continuidades están aseguradas: la ascendente por la pedagogía del príncipe y la descendente por la policía. Más allá de esta doble continuidad, el eje que atraviesa y problematiza la forma de gobierno referirá, según Foucault, a cómo introducir la economía en la política; en la medida que gobernar un Estado será, entonces, poner en movimiento la economía en el nivel del Estado entero (los habitantes, las riquezas, la conducta de todos y de cada uno de los que lo integran).

Si bien la palabra economía refiere en principio al "sabio gobierno de la casa para el bien común de toda la familia" (ROUSSEAU, 1985, p. 241), ${ }^{3}$ la pregunta que se pondrá en juego es cómo este gobierno de la familia puede ser introducido en el gobierno de un Estado. En este contexto, el término economía se constituye como campo de intervención gubernamental, lo cual produce un pasaje del análisis de las riquezas a la economía política, del análisis de la constitución interna del organismo a la población (intermediario entre el medio y el organismo). El arte del gobierno se ocupará de cómo alojar la economía, o sea, "cómo introducir esta atención meticulosa del padre para con su familia, en la gestión del Estado" (RABINOW, 1986, p. 15). Así, la familia ya no atañe al modelo sino a un segmento de la población. "La familia aparece como en ese carácter instrumental con respecto a la población: surgen entonces las campañas concernientes al matrimonio, las vacunaciones, las inoculaciones" (FOUCAULT, 2009b, p. 132). Es decir, el sabio gobierno que refería a la familia se desbloquea y atraviesa a la población, pues el gobierno pasa a relacionarse con una recta disposición sobre las cosas (hábitos, maneras de actuar, decir y pensar, etc.) y en la que los dispositivos pedagógicos tienen mucho que ver; pues se conforman en relación con la ley, con la norma, con el valor, con un poder en la acción a través de la disciplina, del gobierno, de la biopolítica.

"Gobernar demasiado" no formará la respuesta adecuada al problema del gobierno, y por ello el gobierno tratará con una realidad compleja que escapa al territorio: esa nueva realidad es la sociedad. Este quiebre hace que "las esferas social y política estén muchos menos diferenciadas" (ARENDT, 2008, p. 45). El desplazamiento del territorio conllevan una serie de consecuencias: desaparición del modelo familiar como modelo del gobierno (la familia se convertirá ahora en instrumento del gobierno de las poblaciones), surgimiento de la población como

\footnotetext{
${ }^{3}$ La palabra economía procede de oikos, casa, y de mosmos, ley, y no significa originariamente más que el prudente y legítimo gobierno de la casa, para el bien común de toda la familia. Ver: Rousseau, J. J. Discurso sobre la política económica. Madrid: Tecnos, 1985.
} 
el objeto del gobierno (duración de la vida, salud, higiene), y aparición de un saber propio del gobierno que, en sentido lato, se llamará "economía política". En estos desplazamientos, Arendt (2008, p. 45) establece que:

[...] con el ascenso de la sociedad, esto es, del 'conjunto domestico' (oikia), o de las actividades económicas a la esfera pública, la administración de la casa y todas las materias que anteriormente pertenecían a la esfera privada familiar se han convertido en interés "colectivo".

El nacimiento de la economía política produce que la sociedad disipe a la unidad familiar hasta convertirla en su total sustituta, conjuntamente con un desplazamiento que marca el paso de un arte de gobernar a una ciencia política, y el paso de un régimen dominado por las estructuras de la soberanía a un régimen dominado por las técnicas en torno a la población. En líneas generales, al poner a la población en el centro de la escena, lo que surge a principios del siglo XIX es una consideración distinta de la vida, en la cual la relación entre el poder y el sujeto no será simplemente esta forma de sujeción que le permite al primero quitarle al segundo los bienes, riquezas y, ocasionalmente, su cuerpo; en cambio el poder deberá ejercerse sobre los individuos en tanto que constituyen una especie de entidad biológica que debe ser tomada, considerada y cuidada si se desea utilizar esta población como máquina para producir riquezas y bienes.

La educación de los cuerpos, en su faceta de control del cuerpo y regulación de las poblaciones (biopolítica), no es más que una economía, incluso no sería necesario agregar otras palabras o nociones, por ejemplo, 'economía política del detalle' o 'economía política de los cuerpos'. En la medida en que la educación es ese espacio que se constituye entre el naciente mundo privado y el público, entre el individuo y la sociedad (que es necesario inventar y sostener), es ese dispositivo que articula economía y política, es, literalmente, un aspecto de la economía política. (RODRÍGUEZ-GIMÉNEZ, 2010, p. 218).

Este contexto sienta las bases para respondernos el interrogante sobre la aparición del cuerpo en los planes de estudios, como tecnología de poder no sólo orientada al individuo (tecnología disciplinaria del siglo XVIII) sino a la regularización de la población. El hallazgo de la población en un sentido foucaultiano, es junto al descubrimiento del individuo y del cuerpo adiestrable, el núcleo tecnológico en torno al cual los procedimientos políticos de occidente se han transformado.

A partir, de aquí, el cuerpo ya no existirá como un ente biológico, sino dentro y a través de un sistema político que gestiona su vida para establecer cierto tipo de regulación. Ante el problema del gobierno se comienzó a establecer un proceso de gubernamentalidad, una "superficie de contacto en la que se anuda la manera de conducir a los individuos y la manera como éstos se conducen" 
(FOUCAULT, 2009a, p. 497). Este camino no se reduce a cualquier relación de poder, sino que se vincula a las técnicas de gobierno que sirven de base a la formación del Estado moderno. En efecto, la gubernamentalidad será para el Estado lo que las técnicas de segregación serán para la psiquiatría, lo que las técnicas de disciplina serán para el sistema penal, o lo que la biopolítica será para el hospital (Cf. FOUCAULT, 2009b, p. 146-448). Y lo que la pedagogía será para la educación.

Es una modalidad calculada de intervención sobre el elemento físico, en el cual la educación del cuerpo adopta los códigos, reglas y normas propios de la educación de masas, vinculando un saber específico sobre el cuerpo y aspectos pedagógico-políticos. La pedagogía moderna funcionará, en gran parte, como una forma de gobierno, conjuntamente con otras disciplinas que se van constituyendo en el mismo haz de relaciones. Así, la pedagogía, la medicina, la economía, la salud, el sexo, la higiene, entre otros, se convertirán en cuestión de Estado y funcionará en los procesos biosociológicos que atañen a las masas humanas, definiendo ciertos modos de subjetivación (del sujeto con una historia y en una dimensión ética).

Ahora bien, ¿por qué se pone en escena a la población como campo de realidad? No refiere a un cambio de mentalidad de los dirigentes en que ahora toman a la población como objeto y como problema, sino que fue posible en tanto que se produce "un interrogante sobre la manera de repoblar, [...] con referencia a una mortalidad dramática" (FOUCAULT, 2009b. p. 89): a partir de un acontecimiento -en este caso, la escasez que desembocó en la "mortalidad dramática"-, se establecerá una relación de gobierno con ese acontecimiento. No se buscará cierto disciplinamiento tanto sobre el cuerpo como sobre los espacios, sino que, a partir de la norma de la ciencia, se regulará el acontecimiento. En este marco, el acontecimiento "se entrelaza con los conceptos de diagnóstico y de actualidad" (CASTRO, 2011, p. 26), pues el estado logra "neutralizar la inseguridad social, es decir, actuar eficazmente como reductor de riesgos sociales" (CASTEL, 2004, p. 48).

Este acontecimiento, produce un cambio en la manera en la cual el Estado participa, transforma las tecnologías de gobierno mediante un dispositivo social que pone en acción una forma particular del gobierno con el sujeto. Estas tecnologías, por un lado individualizantes, centradas en el control del cuerpo, y por el otro reguladoras de la vida, se articulan optimizando la vida individual y social, ya que ponen en marcha técnicas de racionalización económica que fundan nuevas instituciones. Los saberes que atañen a adiestrar el cuerpo y encauzarlo, permirán construir instituciones como hospitales, prisiones, escuelas. 
En otras palabras, la población tomada como objeto no refiere a una suma de individualidades que habita el territorio, sino que será producto de una serie de variables: entorno material, clima, leyes, costumbres de la gente, intensidad del comercio, valores morales, reglas de higiene que aseguran la longevidad óptima, entre otras. Esto hace redoblar la apuesta de la pedagogía y específicamente de la pedagogía integralista (educación intelectual, moral y física).

Pero ¿cuáles son los límites del gobierno?, ¿cuál es el principio de verdad que rige el límite?, ¿sobre qué enrejado simbólico o realidad se sostienen estas formas de gobierno?, ¿'gobernar en relación a qué cosas? Ante estos interrogantes será necesario analizar estas nuevas formas de organización social para poder establecer cómo, de acuerdo a qué procedimientos se construyen ciertas prácticas de gobierno, qué efectos poseen para poder establecer las relaciones de podersaber-verdad que atraviesan a la pedagogía

Los tipos de relación de poder que permiero el funcionamiento de los sistemas de tipo feudal, están ligados a los signos, a los impuestos, ya que el poder está situado en el campo de la soberanía, es decir, como instrumento de la administración estatal. En el contexto social, político y económico que se presenta a fines del Siglo XVIII, las distintas formas de poder surgen a partir del siguiente interrogante: ¿cómo será posible el gobierno para que las cosas tomen el rumbo más favorable?. Esto producirá una sociedad ya no basada en los problemas de la legalidad, la ley y el derecho, sino en el de la normalización, que será un modo de funcionamiento de la actualidad. ${ }^{4}$ No es el derecho o la ley, sino lo extrajurídico, el saber que pesa sobre los individuos y atraviesa el cuerpo social. Así, esta limitación que se impone en la práctica gubernamental no va a buscarse por el lado de los derechos naturales, en esa razón procedente de Dios, sino en su interior, hacia el lado de sus objetivos: "Las cosas que deben hacerse y las cosas que no deben hacerse" (FOUCAULT, 2007, p. 28).

\section{Política sobre la vida y dispositivo pedagógico}

La constitución del hombre como objeto de conocimiento, en el haz de relaciones en que aparece la pregunta sobre el gobierno y sobre las mejores maneras de gobernar, implica un desbloqueo epistemológico que permitirá el pasaje del análisis de las riquezas a la economía política y el pasaje de la historia natural a la biología; en la medida en que el problema a resolver serán los procesos biosociológicos de las masas humanas.

\footnotetext{
${ }^{4}$ A modo de ejemplo Foucault (2012, p. 35) dice: "mire lo que pasa hoy en día en la justicia penal de la mayoría de los países de Europa. Cuando hay que ocuparse de un criminal, la cuestión es al punto saber si no está loco, cuáles son los motivos psicológicos por los cuales ha cometido sus crimen, los trastornos que experimentó durante su infancia, las perturbaciones de su medio familiar... Las cosas se psicologizan de inmediato; psicologizarlas, es decir medicalizarlas".
} 
Lo que se produjo en el siglo XVIII y a comienzos del siglo XIX fue toda una serie de transformaciones por las cuales se pasó del señalamiento de los caracteres clasificatorios al análisis interno del organismo, y luego del organismo en su coherencia anatómo funcional a sus relaciones constitutivas o reguladoras con el medio de la vida. (FOUCAULT, 2009b, p. 105).

Este control sobre los cuerpos, no se lleva a cabo sólo por medio de cierta ideología, sino que se ejerce sobre el cuerpo, en la medida que la política toma al cuerpo como la realidad biológica, y por ende a la población. Esta política fue llamada biopolítica, o política en nombre de la vida, "y tiene que ver con la población, y ésta como problema político, como problema a la vez científico y político, como problema biológico y problema de poder" (FOUCAULT, 2010, p. 222).

La población deviene a partir de un quiebre epistemológico que la transforma en un elemento condicionante de la realidad del Estado, y la coloca como objeto técnico susceptible de ser epicentro de toda una serie de políticas económicas y, a su vez, de una política sobre la vida. La transformación de la política en biopolítica indica que, en el nacimiento de la modernidad, la vida natural de los hombres empezará a ser incluida en la esfera del poder estatal, constituyendo al cuerpo viviente en blanco de las estrategias políticas del Estado.

Este haz de relaciones se encuentra en la obligación de repartir el saber en dos tramos que se intrincan: el primero se define por lo que ya se sabía (el peso del cartesianismo, el prestigio de Newton), el segundo por lo que no se sabía aún (la evolución, la especificidad de la vida, la noción de organismo). La biología construye un saber sobre el cuerpo basado en la observación y en un modelo de racionalidad que proporcionan las ciencias físicas. En palabras de Foucault (2008b), es una técnica consecuencia de los aportes de Bacon ${ }^{5}$ y de los perfeccionamientos técnicos que le otorga el microscopio a la observación. Es posible describir la biología como aquella ciencia que organiza las relaciones entre funciones y órganos, en las que los seres vivos están ligados a una representación y se encuentran en un haz de relaciones que parten de su existencia. Foucault (2008b), al indagar sobre la historia de la biología, encuentra que ésta era desconocida antes del siglo XVIII, por la razón de que ni siquiera existía la vida misma, sólo existían los seres vivos observados o definidos desde la historia natural.

Ahora bien, este cambio también será acompañado por un análisis de la producción, por una nueva "economía política" que tendrá como papel principal analizar la relación entre el valor y los precios, los conceptos de organismo y

\footnotetext{
${ }^{5}$ Filósofo de la época que establece la creencia de que cualquier idea científica debe estar apoyada, o derivada de la experiencia u observación.
} 
de organización, y los métodos de la anatomía comparada. Así, la biología y la economía política no se constituirán en el lugar de la historia natural, sino allí donde estos saberes no existían, en aquellos intersticios en los que aparece el hombre. Esto les permitirá definirlo a partir de lo vivo, de la vida en relación con lo que lo rodea, pues para que lo vivo pueda vivir es necesario que haya una organización y un movimiento ininterrumpido entre el aire que respira, el agua que bebe y el alimento que absorbe.

En este sentido, el control sobre la población permitirá enlazar las relaciones de saber-poder a través de técnicas que se aplican sobre la población, sobre la vida biológica de la población. Los problemas de la gubernamentalidad serán los problemas de la regulación, los problemas de la pedagogía. Estas relaciones producen un efecto de gobierno que hace que la población requiera "una educación física capaz de poner "el ejercicio al alcance de todos" -como pedía Lagrange en 1894- para no 'abandonar a la gran mayoría, 'a todas las miserias físicas y morales que se derivan de la falta de ejercicio"' (CRISORIO, 2010, p. 53).

De esta manera, los ejercicios físicos de los programas ya no sólo serán, como se enunciaba en el Plan de Estudios de 1903, ejercicios con un criterio absolutamente fisiológico, con repetición mecánica de pasos científicos predeterminados, sino que se redoblará la apuesta: además del control individual del individuo mediante el método racional para el desarrollo natural, en el problema de la vida aparecerá una forma de vida calificada. La ciencia moderna ha jugado un papel fundamental en el tipo de experiencia a partir de la cual el sujeto se construye como objeto de cierta normalidad. Pues en el surgimiento de las artes de gobernar comenzará a regir cierta universalidad del sujeto: la vida en el mundo ya no se considerará como lo que fue, y comenzará a girar sobre la idea de quienes debemos ser, como debe ser esa vida. Como establece Arendt (2008, p. 51),

La sociedad espera de cada uno de sus miembros una cierta clase de conducta, mediante la imposición de innumerables y variadas normas, todas las cuales tienden a 'normalizar' a sus miembros, a hacerlos actuar, a excluir la acción en el marco social.

Aquí, en el objetivo y el límite del gobierno, se define el ser y un deber, en relación a una verdad que se define por las condiciones históricas de posibilidad y que opera por la lucha del sentido. Pero este sentido buscado atañe al cuerpo y, por ende, a la educación, en tanto que ésta se constituye en un dispositivo de las políticas de estado. Si entendemos por dispositivo "la red de relaciones que se pueden tejer entre elementos heterogéneos: discursos, instituciones, arquitectura, reglamento" (CASTRO, 2011, p. 114), la escuela permite establecer el nexo con un discurso sobre la vida y el deber ser de la vida. 
La biología y la medicina sentaron las bases de un saber para definir el cuerpo: un ser orgánico ligado a las condiciones de existencia propias de la representación, a las relaciones entre funciones y órganos y a un concepto sobre la vida que se constituye a partir de una manera de percibir la norma que establece el saber de la medicina (deber ser).

En este auge del cuidado de la vida queda al descubierto un desplazamiento hacia un deber ser, hacia una forma de vida, ya que la vida en tanto que materia física se encuentra apropiada por el poder. No obstante, agrega Esposito (2005, p. 160), esta vida calificada es sobre "toda la vida y sólo la vida, en su simple realidad biológica". Si bien para el autor la idea de bios, significa "vida calificada" o "forma de vida"; la biopolítica remite a la dimensión de la zoé (la vida en su simple mantenimiento biológico); pues, en definitiva, no hace otra cosa que poner a salvo el cuerpo en tanto vida biológica de las poblaciones (la especie), que, "para ser cuerpo, debe mantenerse con vida" (ESPOSITO, 2005, p. 161).

La relación que establece la política con el cuerpo es en el orden de lo viviente, en el orden de la substancia, en tanto que es necesaria alguna representación de tipo orgánico que la enlace con la realidad. Es decir, que para pensar lo bíos, es necesario primero lo biológico y segundo la política. Esto implica pensar que para comprender el cuerpo, primero es necesaria la materia y luego la política Un extracto del Diseño Curricular enuncia una manera de entender el cuerpo en el sentido biopolítico: "El cuerpo constituye una dimensión significativa de la condición humana. No hay vida posible si no es a través del cuerpo y sin su construcción permanente a lo largo de la existencia. Esta construcción es simultáneamente orgánica y subjetiva [...]" (DGCyE, 2006b, p. 131). Es decir, paralelamente se establece la sustancia y la cultura o el lenguaje. Lo mismo cuando afirma que "no hay vida posible, si no es a través del cuerpo" (DGCyE, 2006b, p. 133). Por todo esto, la Educación Secundaria queda reducida a una serie de "experiencias pedagógicas potentes y profundas en el acceso al conocimiento de las artes, la literatura, las ciencias y otros campos de conocimiento" (DGCyE, 2006a, p. 12) reducidas a un acto empirista, a una seria de transformaciones bio-físicas. La biopolítica fundamenta el modo de pensar el cuerpo a partir de la sustancia y en un tiempo y espacio determinado, mediante una práctica pedagógica que normaliza e individualiza.

En Seguridad, territorio y población, Foucault precisa la idea de normalización a partir de la distinción entre la relación disciplina-norma y la relación normadispositivo biopolítico de seguridad. En el primer caso, que será el paradigma que adoptará la pedagogía integralista, se plantea ante todo un modelo óptimo que se construye en función de un determinado resultado (cuerpo dócil, disciplinado, fuerte), y al cual la acción de normalización disciplinaria trata de conformar los gestos y los actos. Es decir lo que hace a la normalización pedagógica o 
disciplinaria no es lo normal y lo anormal, sino la norma que tiene un carácter prescriptivo.

Ahora, al contrario, habrá un señalamiento de lo normal y lo anormal, un señalamiento de las diferentes curvas de normalidad, y la operación de normalización consistirá en hacer interactuar esas diferentes atribuciones de normalidad y procurar que las más desfavorables se asimilen a las más favorables. Tenemos entonces algo que parte de lo normal y se vale de ciertas distribuciones consideradas, para decirlo de alguna manera, como más normales o, en todo caso, más favorables que otras. $\mathrm{Y}$ esas distribuciones servirán de norma. La norma es un juego dentro de las normalidades diferenciales. Lo normal es lo primero y la norma se deduce de ello, o se fija y cumple su papel operativo a partir del estudio de las normalidades. Por consiguiente, $[\ldots]$ ya no se trata de una normación sino más bien, o en sentido estricto, de una normalización. (FOUCAULT, 2009b, p. 83-84).

De este modo, se transforma la función política de las ciencias humanas, que ya no responderán estrictamente a un saber de la normación sino de la normalización y, por lo tanto, no sólo deberán delimitar la norma de lo humano sino incluir, a través de sus discursos, la normalidad de lo humano en los dispositivos de seguridad. Estas condiciones de posibilidad de lo humano constituyen lo humano y forma el telón de fondo del humanismo moderno. El hombre es definido a partir de las relaciones de la biología, la economía y la política. La pedagogía está destinada a ejercer una acción sobre el cuerpo, "para educar y reeducar en la escuela, para participar en la formación de los seres humanos y grupos sociales (NARODOWSKI, 1994, p. 23-24). Funciona como "instancia de delimitación, como el discurso ordenador de lo que puede nombrarse, pensarse y hacerse" (AISENSTEIN; SCHARAGRODSKY, 2006, p. 20). La acción educativa no sólo buscará la marcha natural del cuerpo, sino también un deber ser que, justamente, tiene una vinculación con las variables que hace a la población (cuestiones de salubridad, sexualidad, moralidad, etc.).

La Educación Física no estuvo exenta de esta política de Estado, y fue tomada para el gobierno de la conducta, para la normalización del cuerpo en relación a un estado de salud. En el Diseño Curricular de Educación Física del Nivel Secundario se manifiesta el dispositivo biopolítico al enunciar que "el desarrollo de la propia corporeidad, la participación en experiencias ludomotrices, gimnásticas, deportivas, expresivas, en diferentes ambientes, con crecientes espacios de participación, permiten [...] el cuidado de la salud" (DGCyE, 2006b, p. 133). En otro extracto, se afirma que las prácticas desarrolladas durante la clase de Educación Física deberán permitir, que los alumnos y las alumnas "conozcan las actividades motrices necesarias para el desarrollo de las capacidades condicionales y coordinativas con base en el principio de salud" (DGCyE, 2006b, p. 134). 
La Educación Física se fundamenta en los principios de la salud para prolongar la vida, para mantener al organismo en un estado de normalidad (no enfermo). No obstante, no pensamos que la Educación Física no deba preocuparse por la salud. No es eso en todo caso lo que se busca plantear, sino ¿qué es la salud?, ¿qué cuerpo es saludable y cuál no?, ¿'sobre qué saber se clasifica ese estado del cuerpo?

El lugar en el cual se forma el saber para vigilar la enfermedad ya no es ese “jardín patológico en el cual Dios había distribuido las especies, es una conciencia médica generalizada [...] ligada a cada existencia individual, pero, asimismo, a la vida colectiva de la nación" (FOUCAULT, 2008c, p. 58). En el viraje del Siglo XVIII, al ponerse en discusión el tema clínico, se producirá una mutación esencial en el saber médico, en tanto que la clínica aparece como una nueva experiencia en la cual la mirada médica no se cierra en el vínculo del enfermo, en su singularidad, sino que va más allá, llegando a las dimensiones de la geografía y de un Estado.

El Estado pedirá a los médicos un control estadístico de la salud donde la topografía médica incluya:

[...] resúmenes minuciosos sobre la región, las habitaciones, las personas, las pasiones dominantes, el vestido, la constitución atmosférica, los productos del suelo, el tiempo de su perfecta madurez y de su cosecha, así como la educación física y moral de los habitantes de la comarca. (FOUCAULT, 2008c, p. 57).

De hecho, ya no bastará sólo el control de los médicos sobre las enfermedades de la población, sino además los ciudadanos deberán estar informados del saber médico. Por ello, "cada práctico deberá redoblar su actividad de vigilancia de un papel de enseñanza, porque la mejor manera de evitar que se propague la enfermedad es, aún, difundir la medicina" (p. 57-58). De esta manera, la medicina pasará a tener una tarea fuertemente política, en la que, por sobre todas las cosas, tendrá el poder de instaurar sobre la vida técnicas positivas que lleven a la salud.

En este contexto, la medicina no será sólo el "corpus" de las técnicas de curación y del saber que éstas requieren, desarrollará también un conocimiento del hombre saludable; es decir, a la vez una experiencia del hombre no enfermo y una definición del hombre modelo. (FOUCAULT, 2008c, p. 62).

En efecto, el saber de la medicina tenderá a otorgar a la distinción entre lo normal y lo patológico el poder de delimitar lo que es desviado, anormal, extraño. Aquí lo extraño se excluye y se imponen unos parámetros de normalidad porque "la medicina define no sólo lo que es normal y no normal, sino en definitiva, lo que es lícito o no lícito, criminal o no criminal, lo que es desenfreno o práctica nociva" (FOUCAULT, 2012, p. 270). 
El saber sobre el que se construye la medicina nos deja ver cómo el discurso no es un conjunto de signos, sino una práctica que está constituida por un conjunto de relaciones que van conformando un tipo de sujeto como objeto de una salud, una higiene, una moral. La cuestión no se reduce a la idea de que la ciencia sólo funciona como una suma de objetos cognoscibles, sino que difunde "una fuerza coercitiva sobre cierta verdad, no sólo en dirección al pasado sino al futuro de la humanidad" (FOUCAULT, 2012, p. 92). El saber de la medicina muestra que la vida política se basa en la exclusión de aquello que, se supone, no puede considerarse como normal, colocando lo humano en una normalidad.

[...] el vínculo de la salud con la virtud ya no pasa [...], por la inmediatez natural, sino por el universal dominio de la razón. La salud es el reverso visible de una existencia en la que la totalidad orgánica está determinada, sin oposición ni residuo, por una forma de racionalidad que, más allá de toda partición, es al mismo tiempo ética y orgánica. (FOUCAULT, 2009b, p. 63).

Estas relaciones entre el saber, el poder y la verdad nos permiten ver el dominio de instituciones, los procesos políticos y económicos sobre los cuales se articula el Diseño Curricular de Educación Física con el discurso médico, y nos llevan a pensar una educación que tiene por objetivo remediar la enfermedad o hacer que no aparezca. El pensamiento médico implica:

una manera de percibir las cosas alrededor de la norma, esto es, que procura deslindar lo que es normal de lo que es anormal, que no son justamente lo lícito y lo ilícito [...] busca también asignarse medios de corrección que no con exactamente medios de castigos, sino medios de transformación del individuo, toda una tecnología de comportamiento del ser humano que está ligada a ese fin. (FOUCAULT, 2012, p. 36).

Aquí el cuerpo se sostiene desde la óptica anatomo-fisiológica y patológica, en una caracterización de órganos, sustancias y estados, ya que la esencia saludable de lo humano radica en nuestra condición de individuos, sostenida en lo que sería el interior de nuestro cuerpo biológico. El cuerpo pasa a ser una materialidad compleja y la población la suma de individuos controlados por la tasa de mortalidad, natalidad, entre otra. En términos estrictos, esto lleva a la Educación Física a un dominio de técnicas, a saber hacer en un espacio de aplicación para el desarrollo del organismo; el cual debe llegar a un "principio de salud", a un estado normal a través del desarrollo de "la formación de hábitos de vida saludable" (DGCyE, 2010, p. 9).

Probablemente, la Educación Física deba enseñar, de algún modo, las técnicas necesarias para toda una clase de relaciones (consigo mismo, con los otros, con el saber, etc.); pero ¿por qué con base en el principio de salud? La experiencia queda reducida a la actividad física, a un código orgánico a desarrollar. 
En la descripción del Eje Corporeidad y Motricidad del Diseño Curricular, se incluyen núcleos sintéticos de contenidos, relacionados con la constitución corporal, donde una vez más aparece un cuerpo pensado en órganos y sistemas. $\mathrm{Al}$ respecto se establece que, "los contenidos incluidos refieren al desarrollo integrado de las capacidades motoras, considerando sus aspectos orgánicos, su relación con la salud y la disponibilidad de sí mismo, en un contexto social y cultural de referencia" (DGCyE, 2006b, p. 135).

Y luego, en el apartado de los contenidos enuncia:

Capacidades condicionales:

- La resistencia aeróbica general.

- La fuerza rápida y su desarrollo en los grandes grupos musculares.

- La flexibilidad general.

- La velocidad de desplazamientos en trayectos cortos y variados.

[...] El principio de salud como orientador básico para la realización de tareas motrices. (DGCyE, 2006b, p. 150).

Estos extractos del documento nos permiten ver, por un lado el nexo que se establece entre las medidas administrativas y los enunciados científicos, y por el otro lado la relación entre biopolítica y sistema educativo, en tanto que la regulación del Estado para alargar y normalizar la vida es un elemento que puede justificar la Educación Física en el Sistema Educativo.

En otras palabras, el desarrollo del organismo en relación a una sujeto universal, "normal", "higiénico", "saludable", se da a través de contenidos que "refieren al desarrollo integrado de las capacidades motoras, considerando sus aspectos orgánicos, su relación con la salud” (DGCyE, 2006b, p. 135).

$\mathrm{El}$ dispositivo se halla pues siempre inscripto en un juego de poder, pero también siempre ligado a unos campos de saber, que nacen de él pero, asimismo, lo condicionan. Las relaciones que existen entre los enunciados del discurso sobre la salud, los juegos de conceptos, de series teóricas, se sostienen sobre el saber de la ciencia médica, y colocan al cuerpo como realidad biológica que es intervenida médicamente.

En las sociedades actuales, la experiencia de la vida posee una doble reducción, ya que no sólo refiere a cierto estado no enfermo, sino que vincula a una exaltación de la salud. El interés por el cuerpo ya no está localizado solamente en una política sobre la salud, sino que está vinculado al conjunto de instancias orientadas a optimizar la vida.

De esta manera se van conformando la constitución del sujeto a partir del concepto de salud que, además de determinar un estado orgánico, establece mensajes de cómo vivir la vida, una vida que en la actualidad ha agregado nuevas 
características a ese parámetro de normalidad. A través de las advertencias que se observan en el curriculum, hoy la malla de normalidad es mucho más fuerte y apretada, en tanto que ciertos modelos físicos son vendidos como cuerpos saludables. Por ello, la educación debe "brindar oportunidades para la reflexión crítica sobre la propia corporeidad y los modelos corporales mediáticos circulante" (DGCyE, 2006b, p. 135).

Sin embargo, el conocimiento sobre el cuerpo, no es más que la confirmación de ese patrón pedagógico que pone un predominio sobre lo anatomo-fisiológico, alimentando la fantasía del control absoluto del organismo. El Diseño Curricular establece que se debe abordar "la imagen corporal y el impacto de los modelos mediáticos y de la mirada de los otros en su constitución" (DGCyE, 2011, p. 35), pero lo que se espera de esta relación biopolítica-pedagógica no es del orden de la relación cuerpo-enseñanza o cuerpo-educación, sino de lo estrictamente orgánico (RODRÍGUEZ-GIMÉNEZ, 2008, p. 3).

Pero, para que esta tarea escolar pueda ejercerse con mayor eficiencia, fue necesario centrar la preocupación también sobre los procesos cognitivos, momento en el cual irrumpe una segunda configuración "didáctica que se articula a partir de los aportes de la psicología general y especialmente de la psicología del aprendizaje” (BORDOLI, 2013, p. 185). Así lo didáctico y lo pedagógico funcionarían, con su eficiencia sobre lo cognitivo.

El gobierno de una población descansará, en gran parte, en el eficaz gobierno de sí mismo y la educación, al divulgar la descripción científica del orden del mundo y de las cosas, se constituirá en el mapa de lo representable: aquella verdad que, producida con-ciencia, domina la conciencia. (RODRÍGUEZ GIMÉNEZ, 2007b, p. 50).

\section{El cuerpo de la sustancia y de la evolución}

Entramado en el dispositivo pedagógico, el sistema de enseñanza se constituyó como un espacio clave que supuso satisfacer ese "irrefrenable goce delirante de "enseñar todo a todos"' (BEHARES, 2007, p. 2). En sus desarrollos clásicos modernos, la didáctica y la pedagogía han devenido en tecnologías de intervención, en el aprendizaje una y de conducción la otra. El Diseño Curricular define la Educación Física como "propuesta pedagógica“ (DGCyE, 2010, p. 31) que debe "avanzar en una forma de intervención didáctica” (DGCyE, 2010, p. 29). La pedagogía se presenta en el haz de relaciones de las ciencias bio-médicas, como dispositivo de control de lo corporal, en su dimensión biológica. Por su parte, la didáctica se despliega como teoría de la enseñanza constituida en el conjunto de enunciados del saber psicológico, "del orden de un saber sobre el otro o en ocasión de otro que está en proceso de desarrollo" (BEHARES, 2007, p. 4). 
Esta nueva configuración del dispositivo pedagógico en la lógica de la enseñanza estructura los planes de estudio, a partir del conocimiento sobre el sujeto que otorga la psicología como teoría general de la conducta. Los interrogantes que figuran allí se construyeron conforme al cuerpo que debía ser normalizado, y por ello en la relación pedagógica se instala el alumno, y sus características bio-psico-sociales, como centro. Esta forma de pensar el dispositivo escolar incluye todos los elementos de la realidad (lo biológico, lo psicológico y el entorno), y da cuenta de un modo de entender el sujeto en el cual cada carácter biológico, psicológico y del entorno representa un porcentaje. En diferentes planes de estudios se enuncian estas nuevas configuraciones y relaciones entre la enseñanza y el sujeto de la psicología que colocan en otro lugar al conocimiento. Por ejemplo, ya en las clases de Psicología Pedagógica de los Planes y Programas de Estudio para el ciclo superior de magisterio (que correspondía al $4^{\circ}$ y $5^{\circ}$ año) se establece como contenidos del maestro no sólo conocimientos sino también característica del alumno: "Importancia del estudio integral del niño y del adolescente. Las fases del desarrollo psico-físico. La observación y clasificación de niños y adolescentes en función de edades: cronológica mental" (1953, p. 233). Al respecto Borlodi (2013, p. 185, cursivas del autor) dice:

Los objetos de conocimientos consignados como contenidos para enseñar característicos de los diseños anteriores se reformularon sobre la base de las características de los sujetos aprendientes. Del qué enseñar (recortando el cuerpo de las disciplinas) se pasó a las características de quiénes aprenden (proporcionadas por la psicología) a la hora de planificar las estrategias de enseñanza y aprendizaje.

Esta relación del conocimiento con las características del alumno se continúa sosteniendo en el curriculum de la reforma de los ' 90 , en el cual se establece que "los contenidos propios de la Educación Física pueden ser abordados según una concepción integral del hombre, considerando sus dimensiones cognitiva, afectiva, motriz, funcional, relacional y social" (DGCyE, 2001, p. 179). Por ello se enuncia que "los Contenidos básicos Comunes del Área de Educación Física, en la Provincia de Buenos Aires se han organizado a partir de un criterio psicopedagógico" (DGCyE, 2001, p. 179), y que "el aporte de la psicología a la educación enriquecen la educación obligatoria" (DGCyE, 2007, p. 39). Las características del sujeto, aquellas cuantificables, observables, conforman el eje del aprendizaje y reconfiguran las tecnologías sobre el cuerpo.

$\mathrm{Si}$ bien en el curriculum actual se define de un aspecto "político pedagógico" (DGCyE, 2006a) y no uno psico-pedagógico, el aspecto político produce una identificación sobre el sujeto que lo individualiza y vuelve a caer en lo tangible. Por ello se habla de sujeto docente, sujeto pedagógico, sujeto de 
derecho (Cf. DGCyE, 2007). El cuerpo de la Educación Física es el de la física de Newton en el cual primero hay una masa y luego una materia, en la medida en que el cuerpo posee una esencia, propiedad y naturaleza. No hay nada que no se argumente por el organismo: la salud se argumenta por el organismo, la higiene se argumenta por el organismo, el estado madurativo se argumenta por el organismo, y su existencia se argumenta por el organismo. Por ello, no es casual que en el curriculum se materialicen las etapas del cuerpo, específicamente en psicología, a través de contenidos ligados a las características evolutivas. Por ejemplo se enuncia: "Las edades del hombre. El tiempo y los otros. La infancia, la niñez, la pubertad, la adolescencia/juventud, la adultez y la vejez: un recorrido para ser uno mismo" (DGCyE, 2011, p. 21). Esto reduce una forma de concebir el cuerpo ligado a lo real, que responde a la pregunta qué es el sujeto por medio de la sustancia, que identifica la evolución, y una linealidad en el tiempo. Cada etapa es un paso en el desarrollo que consiste en una verdad obsoleta, y las materias son agrupadas recurrentemente, según el desarrollo normal de las facultades del niño. Este sentido del sujeto de la psicología en correspondencia con un tiempo lineal también se enuncia en el curriculum, cuando establece que en la acción educativa, "se desarrollan ideas acerca de la probable evolución del proceso de construcción de los saberes seleccionados" (DGCyE, 2003b, p. 13), así como en su organización a través de objetivos de aprendizaje que abarcan

[...] las descripciones acerca de los logros que se espera de los alumnos al finalizar el año, y constituyen para el docente un referente que orienta su tarea pedagógica al permitirle ayudar a los alumnos a lograr las mejores aproximaciones a los aprendizajes que en estas expectativas se proponen. (DGCyE, 2010, p. 31).

En esta nueva práctica curricular, reconfigurada por la psicología, los procesos de apropiación del conocimiento y los supuestos procesos de aprendizajes, la lógica epistemológica que define el sujeto no cambia, ya que queda impregnada de cierto objetivo humanista. El sujeto no sólo deberá seguir reglas universales de acuerdo a una salud, una moral y una higiene que se definen por el organismo, sino que también tendrá una característica universal de ser, según su desarrollo temporal identificable o sustancializable. El sujeto siempre se define por un porcentaje de sustancia que queda en el cuerpo, y que permite identificarlo o asignarle un objetivo de aprendizaje. Una vez más, la educación no sale de un humanismo porque el sujeto será caracterizable a partir de la sustancia, y constituido como universal a partir de un registro orgánico.

El saber de la psicología forma, no sólo forma un elemento más del dispositivo escolar junto con la biología y la medicina, sino que permitió una serie de reflexiones ligadas a experiencias prácticas que tuvieron un marcado sesgo didáctico (en el formato propio de los psicologismos imperantes). Por 
ejemplo, en un momento se pudo elaborar una enseñanza conductista, producto de las tradiciones racionalista-empiristas-positivistas, y otra constructivista, a través del argumento de la escuela nueva centrada en el niño (el supuesto de que el sujeto es un yo integrado y completo en el marco de un proceso evolutivo que puede ser predecible). El sujeto queda signado en un tiempo predecible y el saber desplegado en forma temporal; es decir ligado a un tiempo cronológico, no a un tiempo lógico que desestabilizaría a las "didácticas de la mediación" (Cf. BEHARES, 2004); que suponen procesos lineales, con posibilidad de control. Estas relaciones quedan reflejadas en el Diseño Curricular al enunciar que, si al transitar el proceso de aprendizaje ocurren "sucesos no previstos al inicio, tales como la emergencia de dificultades, el alcance de logros en tiempos menores, entre otros sucesos [...] [se] exige al docente redefinir los objetivos de aprendizaje que había formulado al comienzo"(DGCyE, 2010, p. 31).

Lo esencial de la producción del conocimiento queda reducida de esta forma a su dimensión más endeble, esa que a duras penas escapa a las dinámicas utilitarias de la ideología [...] de un cierto trabajo metacognitivo, o un manejo volitivo de la dupla docente alumno, distanciada de las condiciones de ignorantia propias del ejercicio científico. (BEHARES, 2011, p. 82).

Este punto es el que se debe problematizar, ya que el curriculum no ha logrado desprenderse de la consistencia, porque el saber de la biología, de la medicina, de la psicología evolutiva, se argumentan por la existencia de la sustancia. El cuerpo es ligado a una linealidad del tiempo que evoluciona, es constante. Pero el cuerpo, no puede ser representado por la sustancia, por su evolución, sino por las maneras de hacer, pensar y decir de las prácticas. Hay un discurso que atraviesa una práctica, hay una batería significante que "ya está allî" (Lacan), antes que el cuerpo y que constituyen un punto nodal para pensar la educación del cuerpo.

Si el Otro y la batería significante "ya están siempre en su lugar", entonces se despliegan dos conclusiones: 1) que están completos -como el término 'batería' lo indica-, inclusive con la falta ineliminable y los imposibles que comportan y 2) que nadie, ni persona ni grupo, pudo haberlos producido" (EIDELSZTEIN, 2012, p. 9).

En el sentido que le otorga Lacan la experiencia que hace al sujeto es un hecho de discurso, porque en el sujeto "[...] no hay más génesis sino de discurso" (EIDELSZTEIN, 1995, p. 19). Más aún, exclama: "No me canso de decir que esa noción de discurso ha de tomarse como vínculo social, fundado en el lenguaje [...]" (EIDELSZTEIN, 1995, p. 26). Esta posición epistemológica nos lleva a establecer que "no hay cuerpo propio, no hay satisfacción ni insatisfacción antes de un lazo discursivo. Y un lazo discursivo implica al menos el sujeto -o sea, el 
punto de interrogación-, la función del Otro y la preexistencia de significantes; todo eso articulado de determinada manera" (EIDELSZTEIN, 2008, p. 13).

Al definir el cuerpo, si bien no negamos una existencia del cuerpo biológico, es posible establecer que este se construye a partir de "una discontinuidad absoluta, un olvido radical de lo biológico en lo discursivo" (EIDELSZTEIN, 2012, p. 25). La aparición del Otro hace que lo anterior biológico sea olvidado. El otro puede ser un compañero o una compañera, un maestro, en cambio el Otro refiere, en el sentido que le otorga Lacan (1994), al orden simbólico que constituye a la cultura y a la sociedad.

Esta perspectiva pone al Otro, al lenguaje, y la batería significante antes que la sustancia, pues "están antes que toda relación subjetiva" (EIDELSZTEIN, 2012 , p. 9). Entonces, en la constitución del sujeto no se pone primero a la sustancia y luego a la política, sino primero a la política y luego a la sustancia. Esto está indicando que la lógica con la que se lee al cuerpo es la que le da cierto estatuto que admite diferenciarlo en la continuidad de la evolución. Pensar al cuerpo desde un planteo creacionista "parte de la nada -creación ex -nihiloy rechaza las ideas de evolución y maduración" (8). La enseñanza no siempre remite a una evolución ni a una sustancia, porque el sujeto no refiere al individuo biológico, ni a la persona (social o histórica).

El Diseño Curricular ha colocado la sustancia por encima del lenguaje, constituyendo no sólo una educación en un tiempo lineal que se desplaza hacia cierta evolución, sino también un modo de pensar al cuerpo a partir de una energía que es interior, propia del organismo. Sin embargo, para el sujeto incluso el hambre y la sed son significantes, no biológicos. Cuando aparece una práctica que satisface o que disgusta, ya no es del mundo de la naturaleza, de la genética, de lo biológico, "pues lo que de ésta provenía en 'estado puro', ha quedado olvidado para siempre" (44). La relación que el sujeto adquiere con estos estados cobra posición en su lugar, en un enlace con el discurso y en relación a todos los otros significantes en la batería del lenguaje.

\section{Conclusiones}

El recorrido a través de un contenido histórico determinado, es hacer una experiencia de lo que somos, de lo que el curriculum hace, "de lo que es no sólo nuestro pasado sino también nuestro presente, una experiencia de nuestra modernidad de tal modo que salgamos de ella transformados" (FOUCAULT, 2013, p. 36).

La educación se desenlaza en una práctica sistemática, regular y recurrente que posee la responsabilidad de la formación de una "sociedad mejor", definida a partir de un deber ser o de una ilusión en la que la empiria del cuerpo puede ser capturada y pueda funcionar según la estructura del dispositivo. 
Mediante este recorrido genealógico arqueológico de la biopolítica en el marco del dispositivo pedagógico, el cuerpo ha sido un factor de individuación, ya sea por el saber biológico que demanda una condición individual a través de huellas dactilares o rasgos genéticos, por la política que demanda una vida zoé, y también por la psicología en la que se sostiene el dispositivo pedagógico que ubica al cuerpo en el tiempo cronológico, por ejemplo las características evolutivas de los adolescentes.

El cuerpo ha sido definido desde la existencia que se realiza por la operatoria de identificar al cuerpo en la mismidad (en su materialidad, esencias) y no en la alteridad (distinción de lo que las cosas son a partir de lo que no son). En efecto, la teoría pedagógica ha tenido por eje a la persona que va a aprender, aquel a quien se le debe transmitir algo, sin que ese algo adquiera importancia en el modo de pensar la educación. De esto se desprende que las teorías pedagógicas son de naturaleza instrumental: forman un deber ser, saludable, higiénico, ciudadano, etc.

Sin embargo lo humano se halla en un olvido radical de la sustancia viva. El cuerpo es causa del discurso, que hace necesario que para conocer el cuerpo y sus condiciones "sea imprescindible algo totalmente distinto del estudio biológico, genético y hormonal de la sustancia viva” (EIDELSZTEIN, 2012, p. 31).

El sujeto está en el entre por ello no es posible asignarle un punto material para aprehenderlo, pues el sujeto no está ni aquí ni allá sino en el medio. En este sentido, el sujeto no es pensado como un punto, no es posible observarlo en una práctica corporal, ya que funciona como un contenido, es determinado por el significante ${ }^{6}$ " "[...] su mensaje es emitido desde el lugar del Otro" (LACAN, 2009 , p. 567). Si hay algo que caracteriza al sujeto es que se construye entre medio de un sistema de relaciones, de prácticas. Más aún, "es el producto de la relación de un significante ante otro significante" (Cf. EIDELSZTEIN, 1995).

Los saberes funcionan como estructuras que permiten determinadas reglas del sentido a partir de las cuales se constituye el curriculum y un modo de entender el sujeto. Es decir, constituyen a las prácticas en general, que tienen por efecto un sujeto. Por ello, se intentó llevar adelante una crítica de lo que esos discursos nos dicen. No se trató de un rechazo sino de una crítica, de un análisis de los límites y de la reflexión sobre ellos; se trató, en definitiva, de "volver a lanzar tan lejos y tan ampliamente como sea posible el trabajo indefinido de la libertad" (FOUCAULT, 1996a, p. 105), en lo que nos es dado como verdad, como un "deber ser".

\footnotetext{
${ }^{6}$ El significante, no refiere al signo en cuanto que el signo es lo que representa algo para alguien, el significante va más allá, es lo que representa un sujeto para otro significante. Véase Lacan, J. (1961-1962), Seminario 9, La identificación.
} 
Sólo se podrá pensar otras relaciones de enseñanza a partir del desplazamiento de una epistemología que supone el origen del sujeto a partir de la vivencia consciente y la sustancia, hacia una epistemología que pone en el origen del sujeto las estructuras del lenguaje y la racionalidad de las prácticas. En este desplazamiento epistemológico, el sujeto de la educación ya no será el alumno, aquel que se supone que no sabe y por lo tanto tiene que adquirir determinado conocimiento luego del proceso de enseñanza, aquel que no tiene cierta característica universal y que debe alcanzarla; el sujeto será quien hace la educación, desplazando al alumno a la condición de objeto. Pensar la problemática curricular a través de un desplazamiento epistemológico implicar pensar una relación con el saber y con el sujeto que no modifica la manera de definirlos, sino que los desnaturaliza, haciendo aparecer otro sujeto y otra educación.

\section{Referencias}

AISENSTEIN, A.; SCHARAGRODSKY, P. Tras las huellas de la educación física escolar Argentina. Cuerpo, género y pedagogía: 1880-1950. Buenos Aires: Prometeo, 2006.

ARENDT, H. La condición humana. Buenos Aires: Paidós, 2008.

ARGENTINA. Ministerio de Educación de la Nación. Dirección general de enseñanza secundaria, normal, especial y superior. Planes y Programas de Estudio. Ciclo Básico, Segundo Ciclo del Bachillerato, Ciclo Superior del Magisterio, Escuelas Normales Regionales, Escuelas Nacionales de Comercio. Buenos Aires, 1953. Disponible en: <http://www.bnm.me.gov.ar/giga1/documentos/EL000725.pdf>. Acceso en: 12 mar. 2014.

BEHARES, L. Didáctica mínima. Los acontecimientos del saber. Montevideo: Psicolibros, 2004.

BEHARES, L. Enseñar en cuerpo y alma: la teoría de la enseñanza y el saber en la pulsión. Educação Temática Digital, Campinas, v. 8, n. esp., p. 1-21, jun. 2007.

BEHARES, L. Enseñanza y producción de conocimiento. La noción de enseñanza en las políticas universitarias uruguayas. Uruguay: Universidad de la República, 2011.

BORDOLI, E. Revisitando la enseñanza y lo curricular. Apuntes para pensar la igualdad. In: SOUTHWELL, M.; ROMANO, A. (Orgs.). La escuela y lo justo. Ensayos acerca de la medida de lo posible. Buenos Aires: UNIPE, 2013. p. 179-211.

CASTEL, R. La inseguridad social. ¿Qué es estar protegido? Buenos Aires: Manatial, 2004.

CASTRO, E. Diccionario Foucault. Temas, conceptos y autores. Buenos Aires: Siglo XXI, 2011.

CRISORIO, R. Homero y Platón: dos paradigmas de la educación corporal. 2010. 320 f. Tesis (Doctorado en Ciencias de la Educación) - Facultad de Humanidades y Ciencias de la Educación, Universidad Nacional de La Plata, La Plata, 2010.

DIRECCIÓN GENERAL DE CULTURA Y EDUCACIÓN [DGCyE]. Diseño Curricular. Educación Inicial. Educación General Básica. Tomo II. Buenos Aires: Emede S.A., 2001. 
DIRECCIÓN GENERAL DE CULTURA Y EDUCACIÓN [DGCyE]. Documento de Base. En: Programa para la definición del Diseño Curricular del Nivel Polimodal, 2003a. p. 15-21.

DIRECCIÓN GENERAL DE CULTURA Y EDUCACIÓN [DGCYE]. Hacia una mejor educación física en la escuela. Programa: Hacia el fortalecimiento y jerarquización de la educación física como área curricular. Buenos Aires: Talleres Gráficos de la Dirección General de Cultura y Educación de la Provincia de Buenos Aires, 2003b.

DIRECCIÓN GENERAL DE CULTURA Y EDUCACIÓN [DGCyE]. Marco general para la educación secundaria. En: Diseño Curricular para la Educación Secundaria: $1^{\circ}$ año ESB. La Plata, 2006a.

DIRECCIÓN GENERAL DE CULTURA Y EDUCACIÓN [DGCyE]. Educación Física. En: Diseño Curricular para la Educación Secundaria. La Plata, 2006b.

DIRECCIÓN GENERAL DE CULTURA Y EDUCACIÓN [DGCyE]. Marco General de Política Curricular. La Plata, 2007.

DIRECCIÓN GENERAL DE CULTURA Y EDUCACIÓN [DGCyE]. Diseño Curricular para la Educación Secundaria Ciclo Superior. ES4: Educación Física. La Plata, 2010.

DIRECCIÓN GENERAL DE CULTURA Y EDUCACIÓN [DGCyE]. Diseño Curricular para la Educación Secundaria $\mathbf{6}^{\mathbf{0}}$ año: Orientación Educación Física. La Plata, 2011.

ESPOSITO, R. Immunitas. Protección y negación de la vida. Buenos Aires: Amorrortu, 2005.

ESPOSITO, R. Communitas. Buenos Aires: Amorrortu, 2007.

EIDELSZTEIN, A. La estructura del lenguaje: necesidad, demanda y deseo. En: El grafo del deseo. Buenos Aires: Manantial, 1995. p. 42-61.

EIDELSZTEIN, A. El origen del sujeto en psicoanálisis. Revista para el psicoanálisis por venir, La Plata, v. 4, n. 5, p. 7-56, 2002.

EIDELSZTEIN, A. El psicoanálisis entre el otro, el sexo, el amor y el goce. Curso de Posgrado UBA. Clase $\mathrm{N}^{0}$ 2. Buenos Aires, 2008. Disponible en: <http://www. eidelszteinalfredo.com.ar/>. Acceso en: 18 mar. 2014.

EIDELSZTEIN, A. El origen del sujeto en psicoanálisis. Del Big Bang del lenguaje y el discurso en la causación del sujeto. El Rey está desnudo. Revista para el psicoanálisis por venir, Buenos Aires, v. 4, n. 5, p. 7- 55, 2012.

FOUCAULT, M. Microfísica del poder. Madrid: La Piqueta, 1992.

FOUCAULT, M. ¿Qué es la ilustración? Madrid: Ediciones La Piqueta, 1996.

FOUCAULT, M. Historia de la sexualidad 2: la voluntad de saber. Buenos Aires: Siglo XIX, 2005.

FOUCAULT, M. Nacimiento de la biopolítica. Buenos Aires: Fondo de Cultura económico, 2007.

FOUCAULT, M. La arqueología del saber. Buenos Aires: Siglo XXI, 2008a.

FOUCAULT, M. Las palabras y las cosas: una arqueología de las ciencias humanas. Buenos Aires: Siglo XXI, 2008b. 
FOUCAULT, M. El nacimiento de la clínica: una arqueología de la mirada médica. Buenos Aires: Siglo XXI, 2008c.

FOUCAULT, M. La hermenéutica del sujeto. Buenos Aires: Fondo de Cultura Económica, 2009a.

FOUCAULT, M. Seguridad, territorio y población. Buenos Aires: Fondo de Cultura Económica, 2009b.

FOUCAULT, M. Defender la sociedad. Buenos Aires: Fondo de Cultura Económica, 2010.

FOUCAULT, M. El poder una bestia magnifica: sobre el poder, la prisión y la vida. Buenos Aires: Siglo XXI, 2012.

FOUCAULT, M. La inquietud por la verdad: escritos sobre la sexualidad y el sujeto. Buenos Aires: Siglo XXI, 2013.

KANT, I. Pedagogía. Madrid: Akal, 2003.

LACAN, J. Función y campo de la palabra y el lenguaje en psicoanálisis. En: LACAN, J. Escritos I. 17. ed. México: Siglo XXI, 1994. p. 227-310.

LACAN, J. E1 Seminario. 20. ed. Buenos Aires: Paidós, 1995.

LACAN, J. Escritos 2. Buenos Aires: Siglo XXI, 2009.

LARROSA, J. Escuela poder y subjetivación. Madrid: La Piqueta, 1995.

NARODOWSKI, M. Infancia y poder: la conformación de la pedagogía moderna. Buenos Aire: Aique, 1994.

RABINNOW, P. The Foucault reader. Londres: Peregrine, 1986.

RODRÍGUEZ GIMÉNEZ, R. Cuerpo, lenguaje y enseñanza. Educação Temática Digital, Campinas, v. 8, n. esp. P. 31-47, jun. 2007.

RODRIGUEZ-GIMENEZ, R. El cuerpo como objeto de políticas educativas. En: JORNADAS DE CUERPO Y CULTURA UNLP, 2008, La Plata. Anais... La Plata: Facultad Humanidades y Ciencias de la Educación, 2008. Disponible en: < http://www.memoria. fahce.unlp.edu.ar/trab_eventos/ev.633/ev.633.pdf>. Acceso en: 12 mar. 2014.

Rodríguez-Giménez, R. Notas para una investigación sobre pedagogía y biopolítica. Revista Educación física y deporte, Colombia, n. 29-1, p. 215-223, 2010. Disponible en: $<$ http://aprendeenlinea.udea.edu.co/revistas/index.php/educacionfisicaydeporte/article/ viewFile/8497/8027>. Acceso en: 18 mar. 2014.

ROUSSEAU, J. Discurso sobre la economía política. Madrid: Tecnos, 1985.

TIRAMONTI, G. La trama de la desigualdad educativa: mutaciones recientes en la escuela media. Buenos Aires: Manantial, 2007.

Recebido em 30/11/2013

Versão final recebida em 15/06/2014

Aceito em: 10/08/2014 